\title{
SOLUCIÓN LOCAL PARA UNA CLASE DE ECUACIONES NO-LINEALES DEGENERADAS ASOCIADAS A LA ECUACION DE KIRCHHOFF-CARRIER
}

\section{Raúl Izaguirre Maguiña}

RESUMEN.- Sea A el operador definido por la terna $\{H, V,(())$,$\} , donde H, \quad V$, son espacios de Hilbert, la inmersión de $V$ en $H$ es densa y compacta. Consideramos el problema :

$$
\begin{aligned}
& u^{\prime \prime}(t)+M\left(\left|A^{\alpha} u(t)\right|^{2},\left|A^{\beta} u^{\prime}(t)\right|^{2}\right) A^{\gamma} u(t)=f(t) \\
& u(0)=u_{0} \quad ; \quad u^{\prime}(0)=u_{1}
\end{aligned}
$$

donde $M(s, r)$ es una función real derivable en las dos variables y no negativa. Se demuestra la existencia y unicidad de la solución local para $\alpha$, número real positivo y $\beta, \gamma$ números reales no negativos.

\section{INTRODUCTION}

Un sistema propuesto por Lions [ 10 ] para estudiar un modelo desarrollado por Kirchoff, en el año de 1895, sobre las vibraciones de pequeña amplitud de una cuerda fija en sus extremos y cuando la dependencia de la tensión no puede dejarse de lado en el modelo, es dado por

$$
\begin{aligned}
& u^{\prime \prime}(t)+M\left(\left|A^{1 / 2} u(t)\right|^{2}\right) \quad A u(t)=f(t) \\
& u(0)=u_{0} \quad ; \quad u^{\prime}(0)=u_{1}
\end{aligned}
$$

donde, $A$ representa un operador auto-adjunto y positivo de un espacio de Hilbert $H$ y $A^{1 / 2}$ representa su raíz cuadrada. 
Variantes importantes de la Ecuación Abstracta (1) son por ejemplo

$$
\begin{aligned}
& u^{\prime \prime}(t)+M\left(\left|A^{\alpha} u(t)\right|^{2}\right) A^{\beta} u(t)=f(t) \\
& u^{\prime \prime}(t)+M\left(\left|A^{1 / 2} u(t)\right|^{2}\right) A u(t)+A^{\alpha} u^{\prime}(t)=f(t)
\end{aligned}
$$

Para diversos valores de los exponentes $\alpha$ y $\beta$, la ecuación (2) permite estudiar ecuaciones del tipo Kirchhoff-Carrier, ver por ejemplo Arosio-Spagnolo [2], Cousin-Frota, Larkin-Medeiros [4], Crippa [5], Ebihara-Medeiros-Milla [6], Izaguirre-Véliz [8], Medeiros-Milla [11], Pohozaev [18], [19], Perla [17], Rivera [20] y Yamada [21].

Para el caso de la ecuacion (3) considerar Alves - Oliveira [1], Ikehata [7], Medeiros - Milla[12], Nakao - Ono [13], Nishihara - Yamada [14] y Ono [15], [16] .

Nosotros estudiamos en el presente trabajo un modelo de la forma

$$
u^{\prime \prime}(t)+M\left(\left|A^{\alpha} u(t)\right|^{2},\left|A^{\beta} u^{\prime}(t)\right|^{2}\right) A^{\gamma} u(t)=f(t)
$$

donde la función no-lineal $M(s, r)$ es no negativa de clase $\mathrm{C}^{1}$ en las dos variables.

\section{PRELIMINARES}

Sean $(V, a(u, v)),(H,(u, v))$ espacios de Hilbert, $V \subset H$, la inmersión de $V$ en $H$ es densa y compacta. Sea también $A$, el operador definido por la terna $\{V, H, a(u, v)\}$. Entonces, $D(A)$ es un subespacio denso en $H ; A$ es un operador no acotado auto adjunto y positivo de $H$,con espectro discreto;

$$
A w_{v}=\lambda_{v} w_{v} \quad ; 0<\lambda_{1} \leq \lambda_{2} \leq \ldots \leq \lambda v \leq \ldots \quad ; \quad \lambda_{v} \stackrel{v \rightarrow \infty}{\longrightarrow} \infty
$$

$\left\{w_{v}\right\}_{v \geq 1}$ es un sistema ortonormal completo de $H$ de modo que

$$
\begin{aligned}
& D(A)=\left\{u \in H ; \sum_{v=1}^{\infty} \lambda_{v}^{2}\left|\left(u, w_{v}\right)\right|^{2}<\infty\right\} \\
& A u=\sum_{v=1}^{\infty} \lambda_{v}\left(u, w_{v}\right) w_{v} \quad \forall u \in D(A)
\end{aligned}
$$

Asímismo, para todo $\alpha>0$, el operador $A^{\alpha}$ está bien definido por 


$$
\begin{aligned}
& D\left(A^{\alpha}\right)=\left\{u \in H ; \sum_{v=1}^{\infty} \lambda_{v}^{2 \alpha}\left|\left(u, w_{v}\right)\right|^{2}<\infty\right\} \\
& A^{\alpha} u=\sum_{v=1}^{\infty} \lambda_{v}^{\alpha}\left(u, w_{v}\right) w_{v} \quad \forall u \in D(A) \\
& |u|_{\alpha}^{2}=\left|A^{\alpha} u\right|^{2}=\sum_{v=1}^{\infty} \lambda_{v}^{2 \alpha}\left|\left(u, w_{v}\right)\right|^{2}
\end{aligned}
$$

se tiene que $\left(D\left(A^{\alpha}\right),|\cdot|_{\alpha}\right)$ es un espacio de Hilbert y si $\alpha<\beta$ la inmersión de $D\left(A^{\beta}\right) \subset D\left(A^{\alpha}\right)$ es compacta $\forall \alpha, \beta \in R$.

\section{Hipótesis Sobre la Función $M$}

H-1 $M \in C^{1}\left(\left[0, T_{0}\right] \times\left[0, T_{0}\right] ; R^{+}\right)$, donde $T_{0}$ es un número real positivo.

H-2 Si $s_{1} \leq s_{2}, \quad r_{1} \leq r_{2}$, entonces $M\left(s_{1}, r_{1}\right) \leq M\left(s_{2}, r_{2}\right)$

H-3 $M(0,0)=0 ;$ Si $s>0$ entonces $M(s, r)>0 ; \forall r \geq 0$

\section{Definición de Constantes}

En esta parte definimos diversas constantes que aparecen en el desarrollo del trabajo.

C-1 $\left|A^{\alpha} v\right| \leq d_{0}\left|A^{\alpha+\beta+\gamma} v\right| \quad \forall v \in D\left(A^{\alpha+\beta+\gamma}\right)$

C-2 $\left|A^{\beta} v\right| \leq d_{1}\left|A^{\alpha+\beta+\gamma / 2} v\right| \quad \forall v \in D\left(A^{\alpha+\beta+\gamma / 2}\right)$

C-3 $\left|A^{\alpha+\beta+\gamma / 2} v\right| \leq d_{2}\left|A^{\alpha+\beta+\gamma} v\right| \quad \forall v \in D\left(A^{\alpha+\beta+\gamma}\right)$

C-4 $\left|A^{\beta+\gamma} v\right| \leq d_{3}\left|A^{\alpha+\beta+\gamma} v\right| \quad \forall v \in D\left(A^{\alpha+\beta+\gamma}\right)$

C-5 $\left|A^{\alpha+\beta+\gamma} v\right| \leq d_{4}\left|A^{\alpha+\beta+3 \gamma / 2} v\right| \quad \forall v \in D\left(A^{\alpha+\beta+3 \gamma / 2}\right)$

C-6 $\quad a_{1}=M\left(d_{0}^{2} k^{2}, d_{1}^{2} k^{2}\right)$

C-7 Desde que por continuidad $M(s, r) \rightarrow 0$ si $(s, r) \rightarrow(0,0)$, podemos elegir un $k$ suficientemente pequeño tal que

$$
a_{1}^{2} \leq 1 / 6 d_{1}^{2}
$$

Para $k$ seleccionado de esta forma consideramos: 
C-8 $\quad b_{1}=\operatorname{Sup}\left|M_{s}(s, r)\right| \quad \forall \quad s, r \in\left[0, d_{0}^{2} k^{2}\right] \times\left[0, d_{1}^{2} k^{2}\right]$

C-9 $b_{2}=\operatorname{Sup}\left|M_{r}(s, r)\right| \quad \forall \quad s, r \in\left[0, d_{0}^{2} k^{2}\right] \times\left[0, d_{1}^{2} k^{2}\right]$

C-10 $b^{*}=2 d_{1}\left\{b_{1} d_{0}+b_{2}\right\}$

Para datos iniciales $0 \neq u_{0}, u_{1}, f$ convenientes consideramos

C-11 $C_{1}=\left|u_{1}\right|_{\alpha+\beta+\gamma}^{2}+a_{1}\left|u_{0}\right|_{\alpha+\beta+3 \gamma / 2}^{2}+|f|_{L^{\infty}\left(0, T_{0} ; D\left(A^{\alpha+\beta+\gamma}\right)\right)}^{2}$

Por la H-3, podemos definir

C-12 $0<m_{0}=M\left(\frac{\left|A^{\alpha} u_{0}\right|^{2}}{4}, 0\right)$

C-13 $d^{*}=\frac{\operatorname{Max}\left\{d_{2}^{2}, d_{4}^{2}\right\}}{\min \left\{1, m_{0}\right\}}$

C-14 $\quad T^{*}=\frac{\left|A^{\alpha} u_{0}\right|}{2 d_{0} k}$

C-15 $T_{0}=\min \left\{T^{*}, \frac{1}{b^{*} k^{2}}\right\}$

Consideremos el siguiente conjunto

$$
G=\left\{\begin{array}{l}
v \in L^{\infty}\left(0, T_{0} ; D\left(A^{\alpha+\beta+\gamma}\right)\right) / v^{\prime} \in L^{\infty}\left(0, T_{0} ; D\left(A^{\alpha+\beta+\gamma / 2}\right)\right), v^{\prime \prime} \in L^{\infty}\left(0, T_{0} ; D\left(A^{\beta}\right)\right) \\
v(0)=u_{0} \neq 0 ; v^{\prime}(0)=u_{1} ;\left|A^{\beta} v^{\prime \prime}(t)\right|^{2}+\left|A^{\alpha+\beta+\gamma / 2} v^{\prime}(t)\right|^{2}+\left|A^{\alpha+\beta+\gamma} v(t)\right|^{2} \leq k^{2} \quad \forall t \in\left[0, T_{0}\right]
\end{array}\right\}
$$

Lema 1. Sí $v \in G$ y $\psi(t)=M\left(\left|A^{\alpha} v(t)\right|^{2},\left|A^{\beta} v^{\prime}(t)\right|^{2}\right)$,

entonces

(1) $0<m_{0} \leq \psi(t) \quad \forall t \in\left[0, T^{*}\right]$

(2) $\left|\psi^{\prime}(t)\right| \leq b^{*} k^{2}$

Demostración. Sea $v \in G$, entonces

$$
\| A^{\alpha} v(t)|-| A^{\alpha} v(0)|| \leq\left|A^{\alpha} v(t)-A^{\alpha} v(0)\right|=\left|\int_{0}^{t} A^{\alpha} v^{\prime}(s) d s\right| \leq \int_{0}^{t}\left|A^{\alpha} v^{\prime}(s)\right| d s \leq d_{0} t k
$$


De esta desigualdad obtenemos

$$
\left|A^{\alpha} u_{0}\right|-d_{0} k t=\left|A^{\alpha} v(0)\right|-d_{0} k t \leq\left|A^{\alpha} v(t)\right|
$$

Luego;

$$
0<\frac{\left|A^{\alpha} u_{0}\right|}{2} \leq\left|A^{\alpha} u_{0}\right|-d_{0} k T_{1}^{*} \leq\left|A^{\alpha} u_{0}\right|-d_{0} k t \leq\left|A^{\alpha} v(t)\right| \quad \forall t \in\left[0, T^{*}\right]
$$

Por la hipótesis $\mathrm{H}-2$

$$
\psi(t)=M\left(\left|A^{\alpha} u(t)\right|^{2},\left|A^{\beta} u^{\prime}(t)\right|^{2}\right) \geq M\left(\frac{\left|A^{\alpha} u_{0}\right|^{2}}{4}, 0\right)=m_{0}>0
$$

lo que demuestra (1).

Debemos ahora obtener estimativas para $\psi^{\prime}(t)$. Tenemos que

$$
\begin{aligned}
\psi^{\prime}(t)= & 2 M_{s}\left(\left|A^{\alpha} v(t)\right|^{2},\left|A^{\beta} v^{\prime}(t)\right|^{2}\right)\left(A^{\alpha} v(t), A^{\alpha} v^{\prime}(t)\right)+ \\
& +2 M_{r}\left(\left|A^{\alpha} v(t)\right|^{2},\left|A^{\beta} v^{\prime}(t)\right|^{2}\right)\left(A^{\beta} v^{\prime}(t), A^{\beta} v^{\prime \prime}(t)\right)
\end{aligned}
$$

donde $M_{s}, M_{r}$, representan las derivadas parciales con respecto a las variables $s$ y $r$.

Entonces,

$$
\begin{aligned}
\left|\psi^{\prime}(t)\right| & \leq 2\left|M_{s}\left(\left|A^{\alpha} v(t)\right|^{2},\left|A^{\beta} v^{\prime}(t)\right|^{2}\right)\left(A^{\alpha} v(t), A^{\alpha} v^{\prime}(t)\right)\right|+ \\
& +2\left|M_{r}\left(\left|A^{\alpha} v(t)\right|^{2},\left|A^{\beta} v^{\prime}(t)\right|^{2}\right)\left(A^{\beta} v^{\prime}(t), A^{\beta} v^{\prime \prime}(t)\right)\right| \leq \\
& \leq 2 b_{1} d_{0} d_{1} k^{2}+2 b_{2} d_{1} k^{2}=b^{*} k^{2}
\end{aligned}
$$

Problema Lineal. Sea entonces $v \in G$. Nos planteamos resolver el problema siguiente

$$
\begin{aligned}
& u^{\prime \prime}+M\left(\left|A^{\alpha} v(t)\right|^{2},\left|A^{\beta} v^{\prime}(t)\right|^{2}\right) A^{\gamma} u=f \\
& u(0)=u_{0} ; u^{\prime}(0)=u_{1}
\end{aligned}
$$

Teorema 1. Sean

$$
\begin{aligned}
& 0 \neq u_{0} \in D\left(A^{\alpha+\beta+3 \gamma / 2}\right) \\
& u_{1} \in D\left(A^{\alpha+\beta+\gamma}\right) \\
& f \in L^{\infty}\left(0, T_{0} ; D\left(A^{\alpha+\beta+\gamma}\right)\right) ; f^{\prime} \in L^{\infty}\left(0, T_{0} ; D\left(A^{\alpha+\beta+\gamma / 2}\right)\right)
\end{aligned}
$$


que satisfacen las siguientes condiciones

donde

$$
C_{1} \leq k^{2} C_{2}
$$

$$
C_{2}=\min \left\{\frac{1}{3 d^{*} e}, \frac{1}{6 d_{1}^{2} d_{2}^{2} c_{4}^{2}}\right\}
$$

Entonces para todo $v \in G$, existe una única solución $u \in G$ del problema (4) tal que

$$
\begin{aligned}
& u \in L^{\infty}\left(0, T_{0} ; D\left(A^{\alpha+\beta+\gamma}\right)\right) \\
& u^{\prime} \in L^{\infty}\left(0, T_{0} ; D\left(A^{\alpha+\beta+\gamma / 2}\right)\right) \\
& u^{\prime \prime} \in L^{\infty}\left(0, T_{0} ; D\left(A^{\beta}\right)\right) \\
& \left(u^{\prime \prime}(t), z\right)+M\left(\left|A^{\alpha} v(t)\right|^{2},\left|A^{\beta} v^{\prime}(t)\right|^{2}\right)\left(A^{\gamma} u(t), z\right)=(f(t), z) \\
& \forall z \in L^{2}\left(\left[0, T_{0}\right] ; D\left(A^{\alpha+\beta+\gamma}\right)\right)
\end{aligned}
$$

Demostración. Sea $V_{m}=\left[w_{1}, \ldots, w_{m}\right]$, el subespacio generado por los primeros vectores propios del operador $A$. Luego $V_{m}$ es un subespacio de $V$ de dimensión finita $m$, e invariante bajo la acción del operador $A^{\delta}, \forall \delta \in \boldsymbol{R}$, es decir $A^{\delta}\left(V_{m}\right) \subset V_{m}$. Sea entonces

$$
u_{m}(t)=\sum_{i=1}^{m} g_{i m}(t) w_{i} \in V_{m}
$$

Donde las funciones $g_{\text {im }}$ son determinadas por la solución del siguiente sistema de ecuaciones diferenciales ordinarias lineales

$$
\begin{aligned}
& \left(u_{m}^{\prime \prime}(t), w_{j}\right)+M\left(\left|A^{\alpha} v(t)\right|^{2},\left|A^{\beta} v^{\prime}(t)\right|^{2}\right)\left(A^{\gamma} u(t), w_{j}\right)=(f(t), z) \\
& u_{m}(0)=u_{0 m}=\sum_{i=0}^{m}\left(u_{0}, w_{j}\right) w_{j} \stackrel{m \rightarrow 0}{\longrightarrow} u_{0} \\
& u_{m}^{\prime}(0)=u_{1 m}=\sum_{i=0}^{m}\left(u_{1}, w_{j}\right) w_{j} \stackrel{m \rightarrow 0}{\longrightarrow} u_{1}
\end{aligned}
$$

Luego de un análisis y aplicación del Teorema de Caratheodory sobre existencia local de solución de ecuaciones diferenciales ordinarias lineales este sistema admite solución en un intervalo $\left[0, t_{m}\right)$, de donde se sigue la existencia de las 
soluciones aproximadas $u_{m}$ para $m \geq 1$. Seguidamente, debemos obtener estimativas a priori, para la sucesión $\left\{u_{m}\right\}$ de modo que podamos prolongarlas a un intervalo uniforme de existencia.

\section{ESTIMATIVA A PRIORI I}

Haciendo $w=2 A^{2 \alpha+2 \beta+2 \gamma} u_{m}^{\prime}(t) \in V_{m}$, en la ecuación aproximada (16), obtenemos una relación de la forma

$$
\begin{aligned}
\frac{d}{d t}\left\{\left|A^{\alpha+\beta+\gamma} u_{m}^{\prime}(t)\right|^{2}+\psi(t)\left|A^{\alpha+\beta+3 \gamma / 2} u_{m}(t)\right|^{2}\right\} & =2\left(A^{\alpha+\beta+\gamma} f(t), A^{\alpha+\beta+\gamma} u_{m}^{\prime}(t)\right)+ \\
& +\psi^{\prime}(t)\left|A^{\alpha+\beta+3 \gamma / 2} u_{m}(t)\right|^{2}
\end{aligned}
$$

donde nuevamente

$$
\psi(t)=M\left(\left|A^{\alpha} v(t)\right|^{2},\left|A^{\beta} v^{\prime}(t)\right|^{2}\right)
$$

Por el Lema 1, obtenemos

$$
\begin{aligned}
\frac{d}{d t}\left\{\left|A^{\alpha+\beta+\gamma} u_{m}^{\prime}(t)\right|^{2}+\psi(t)\left|A^{\alpha+\beta+3 \gamma / 2} u_{m}(t)\right|^{2}\right\} & \leq\left|A^{\alpha+\beta+\gamma} f(t)\right|^{2}+\left|A^{\alpha+\beta+\gamma} u_{m}^{\prime}(t)\right|^{2}+ \\
& +b^{*} k^{2}\left|A^{\alpha+\beta+3 \gamma / 2} u_{m}(t)\right|^{2}
\end{aligned}
$$

Sea

$$
\varphi(t)=\left|A^{\alpha+\beta+\gamma} u_{m}^{\prime}(t)\right|^{2}+m_{0}\left|A^{\alpha+\beta+3 \gamma / 2} u_{m}(t)\right|^{2}
$$

Entonces, integrando en la ecuación (14) de 0 a $t$, obtenemos

$$
\begin{aligned}
\varphi(t) & \leq\left|A^{\alpha+\beta+\gamma} u_{m}^{\prime}(t)\right|^{2}+\psi(t)\left|A^{\alpha+\beta+3 \gamma / 2} u_{m}(t)\right|^{2} \leq\left|A^{\alpha+\beta+\gamma} u_{1 m}\right|^{2}+\psi(0)\left|A^{\alpha+\beta+3 \gamma / 2} u_{0 m}\right|^{2}+ \\
& +\int_{0}^{t}\left|A^{\alpha+\beta+\gamma} f(s)\right|^{2} d s+\int_{0}^{t}\left|A^{\alpha+\beta+\gamma} u_{m}^{\prime}(s)\right|^{2} d s+b^{*} k^{2} \int_{0}^{t}\left|A^{\alpha+\beta+3 \gamma / 2} u_{m}(s)\right|^{2} d s \\
& \leq\left|A^{\alpha+\beta+\gamma} u_{1}\right|^{2}+\psi(0)\left|A^{\alpha+\beta+3 \gamma / 2} u_{0}\right|^{2}+\int_{0}^{T_{0}}\left|A^{\alpha+\beta+\gamma} f(s)\right|^{2} d s+ \\
& +\int_{0}^{t}\left|A^{\alpha+\beta+\gamma} u_{m}^{\prime}(s)\right|^{2} d s+b^{*} k^{2} \int_{0}^{t}\left|A^{\alpha+\beta+3 \gamma / 2} u_{m}(s)\right|^{2} d s
\end{aligned}
$$

Por lo tanto hemos obtenido una desigualdad de la forma siguiente

$$
\varphi(t) \leq C_{1}+b^{*} k^{2} \int_{0}^{t} \varphi(s) d s
$$


Por el lema de Gronwall concluimos que

$$
\varphi(t) \leq C_{1} e^{b^{*} k^{2} t}
$$

ESTIMATIVA 2. A continuación obtendremos una acotación para la sucesión $\left(u_{m}^{\prime \prime}\right)$. Tenemos que la proyección ortogonal $P_{m}: H \rightarrow V_{m}$ dada por

$$
P_{m}(v)=\sum_{j=1}^{\infty}\left(v, w_{j}\right) w_{j}
$$

es un operador acotado y con norma $\|P\|=1\left\|P_{m}\right\| \leq 1$ y además $P_{m} u=u \quad \forall u \in V_{m}$. Reemplazando $w_{j}$ por $A^{\alpha+\beta+\gamma / 2} w_{j}$ en la ecuación aproximada (16), multiplicando por el vector $w_{j}$ y sumando desde $j=1 a j=m$, obtenemos

$$
\sum_{j=1}^{m}\left(A^{\alpha+\beta+\gamma / 2} u_{m}^{\prime \prime}(t), w_{j}\right) w_{j}+\psi(t) \sum_{j=1}^{m}\left(A^{\alpha+\beta+\gamma / 2} u_{m}(t), w_{j}\right) w_{j}=\sum_{j=1}^{m}\left(A^{\alpha+\beta+\gamma / 2} f(t), w_{j}\right) w_{j}
$$

Analizando cada uno de los términos que aparecen en la ecuación (25).

$$
\begin{aligned}
& \sum_{j=1}^{m}\left(A^{\alpha+\beta+\gamma / 2} u_{m}^{\prime \prime}(t), w_{j}\right) w_{j}=P_{m} A^{\alpha+\beta+\gamma / 2} u_{m}^{\prime \prime}(t)=A^{\alpha+\beta+\gamma / 2} u_{m}^{\prime \prime}(t) \\
& \psi(t) \sum_{j=1}^{m}\left(A^{\alpha+\beta+3 \gamma / 2} u_{m}(t), w_{j}\right) w_{j}=P_{m} A^{\alpha+\beta+3 \gamma / 2} u_{m}(t)=A^{\alpha+\beta+3 \gamma / 2} u_{m}(t) \\
& \sum_{j=1}^{m}\left(A^{\alpha+\beta+\gamma / 2} f(t), w_{j}\right) w_{j}=P_{m} A^{\alpha+\beta+\gamma / 2} f(t)
\end{aligned}
$$

Por lo tanto

$$
A^{\alpha+\beta+\gamma / 2} u_{m}^{\prime \prime}(t)=-\psi(t) A^{\alpha+\beta+3 \gamma / 2} u_{m}(t)+P_{m} A^{\alpha+\beta+\gamma / 2} f(t)
$$

luego

$$
\begin{aligned}
\left|A^{\alpha+\beta+\gamma / 2} u_{m}^{\prime \prime}(t)\right| & \leq \psi(t)\left|A^{\alpha+\beta+\gamma / 2} u_{m}(t)\right|+\left|P_{m} A^{\alpha+\beta+\gamma / 2} f(t)\right| \leq \\
& \leq a_{1} k+C_{4}|f|_{L^{\circ}\left(0, T_{0} ; D\left(A^{\alpha+\beta+\gamma / 2}\right)\right)} \leq \\
& \leq 2\left\{a_{1}^{2} k^{2}+C_{4}^{2}|f|_{\left.L^{\infty}\left(0, T_{0} ; D\left(A^{\alpha+\beta+\gamma / 2}\right)\right)\right\} .}^{2}\right.
\end{aligned}
$$

Teniendo en cuenta las estimativas 1 y 2 deducimos que

$$
\left(u_{m}^{\prime \prime}\right) \text { es acotada en } L^{\infty}\left(0, T_{0} ; D\left(A^{\alpha+\beta+\gamma / 2}\right)\right) \text {. }
$$


ESTIMATIVA 3. Derivando formalmente en la ecuación aproximada con relación a la variable $t$ obtenemos

$$
\left(u_{m}^{\prime \prime \prime}(t), w_{j}\right)+\psi(t)\left(A^{\gamma} u^{\prime}(t), w_{j}\right)+\psi^{\prime}(t)\left(A^{\gamma} u(t), w_{j}\right)=\left(f^{\prime}(t), w_{j}\right) .
$$

Reemplazando en la ecuación (32) $w_{j}$ por $A^{\beta} w_{j}$, multiplicando por el vector $w_{j}$ y sumando de $j=1$ a $j=m$ y procediendo como en la Estimativa 2 obtenemos

$$
A^{\beta} u_{m}^{\prime \prime \prime}(t)=-\psi(t) A^{\beta+\gamma} u_{m}^{\prime}(t)-\psi^{\prime}(t) A^{\beta+\gamma} u_{m}(t)+P_{m} A^{\beta} f^{\prime}(t)
$$

de donde

$$
\begin{aligned}
\left|A^{\beta} u_{m}^{\prime \prime \prime}(t)\right| & \leq \psi(t)\left|A^{\beta+\gamma} u_{m}^{\prime}(t)\right|+\left|\psi^{\prime}(t)\right|\left|A^{\beta+\gamma} u_{m}(t)\right|+\left|P_{m} A^{\beta} f^{\prime}(t)\right| \leq \\
& \leq a_{1} d_{3} k+b^{*} d_{4} k^{3}+d_{1}\left|f^{\prime}\right|_{L^{\infty}\left(0, T_{0} ; D\left(A^{\beta}\right)\right)}
\end{aligned}
$$

Luego :

$$
\left(u_{m}^{\prime \prime \prime}\right) \text { es acotada en } L^{\infty}\left(0, T_{0} ; D\left(A^{\beta}\right)\right)
$$

\section{CONVERGENCIA DE LAS SOLUCIONES APROXIMADAS}

Por las estimativas 1,2 y 3 , tenemos que

$$
\begin{aligned}
& \left(u_{m}\right) \text { es acotada en } L^{\infty}\left(0, T_{0} ; D\left(A^{\alpha+\beta+3 \gamma / 2}\right)\right) \\
& \left(u_{m}^{\prime}\right) \text { es acotada en } L^{\infty}\left(0, T_{0} ; D\left(A^{\alpha+\beta+\gamma}\right)\right) \\
& \left(u_{m}^{\prime \prime}\right) \text { es acotada en } L^{\infty}\left(0, T_{0} ; D\left(A^{\alpha+\beta+\gamma / 2}\right)\right) \\
& \left(u_{m}^{\prime \prime \prime}\right) \text { es acotada en } L^{\infty}\left(0, T_{0} ; D\left(A^{\beta}\right)\right) .
\end{aligned}
$$

Por las inmersiones compactas: $D\left(A^{\alpha+\beta+3 \gamma / 2}\right) \subset D\left(A^{\alpha+\beta+\gamma}\right) \subset D\left(A^{\alpha+\beta+\gamma / 2}\right) \subset D\left(A^{\beta}\right)$ las estimativas 1, 2, 3 y aplicando el Lema de Aubins-Lions, existe una función $u \in L^{\infty}\left(0, T_{0} ; D\left(A^{\alpha+\beta+\gamma}\right)\right)$ tal que,

$$
\begin{aligned}
& u_{m} \stackrel{m \rightarrow \infty}{\longrightarrow} u \text { fuerte en } L^{4}\left(0, T_{0} ; D\left(A^{\alpha+\beta+\gamma}\right)\right) \\
& u_{m}^{\prime} \stackrel{m \rightarrow \infty}{\longrightarrow} u^{\prime} \text { fuerte en } L^{4}\left(0, T_{0} ; D\left(A^{\alpha+\beta+\gamma / 2}\right)\right) \\
& u_{m}^{\prime \prime} \stackrel{m \rightarrow \infty}{\longrightarrow} u^{\prime \prime} \text { fuerte en } L^{2}\left(0, T_{0} ; D\left(A^{\beta}\right)\right)
\end{aligned}
$$

Procediendo de forma estándar obtenemos que la función $u$ es solución fuerte del problema (4) - (5). 
Ahora probaremos que para numeros suficientemente pequeños y una conveniente constante $k$ la solución $u$ del problema (4) pertenece al conjunto G.

En efecto, por (40), (41) y (42) tenemos

$$
\begin{aligned}
& \left|A^{\alpha+\beta+\gamma} u_{m}\right|^{2} \stackrel{{ }_{m \rightarrow \infty}}{\longrightarrow}\left|A^{\alpha+\beta+\gamma} u\right|^{2} \text { fuerte en } L^{2}\left(0, T_{0}\right) \\
& \left|A^{\alpha+\beta+\gamma / 2} u_{m}^{\prime}\right|^{2} \stackrel{m \rightarrow \infty}{\longrightarrow}\left|A^{\alpha+\beta+\gamma / 2} u^{\prime}\right|^{2} \text { fuerte en } L^{2}\left(0, T_{0}\right) \\
& \left|A^{\beta} u_{m}^{\prime \prime}\right|^{2} \stackrel{m \rightarrow \infty}{\longrightarrow}\left|A^{\beta} u^{\prime \prime \prime}\right|^{2} \text { fuerte en } L^{2}\left(0, T_{0}\right) .
\end{aligned}
$$

por otro lado,

$$
\begin{aligned}
\left|A^{\beta} u_{m}^{\prime \prime}\right|^{2}+\left|A^{\alpha+\beta+\gamma / 2} u_{m}^{\prime}\right|^{2}+\left|A^{\alpha+\beta+\gamma} u_{m}\right|^{2} & \leq \\
& \leq d_{1}^{2}\left|A^{\alpha+\beta+\gamma / 2} u_{m}^{\prime \prime}\right|^{2}+d_{2}^{2}\left|A^{\alpha+\beta+\gamma} u_{m}^{\prime}\right|^{2}+d_{4}^{2}\left|A^{\alpha+\beta+3 \gamma / 2} u_{m}\right|^{2} \leq \\
& \leq 2 d_{1}^{2} a_{1}^{2} k^{2}+2 d_{1}^{2} C_{4}^{2}|f|_{L^{2}\left(0, T_{0} ; D\left(A^{\alpha+\beta+\gamma / 2}\right)\right)}^{2}+d^{*} C_{1} e^{b^{*} k^{2} T_{0}}
\end{aligned}
$$

Por las condiciones (C-7), (9), (10) obtenemos

$$
\left|A^{\beta} u_{m}^{\prime \prime}\right|^{2}+\left|A^{\alpha+\beta+\gamma / 2} u_{m}^{\prime}\right|^{2}+\left|A^{\alpha+\beta+\gamma} u_{m}\right|^{2} \leq k^{2}
$$

Entonces pasando al limite, $m \rightarrow \infty$, teniendo en cuenta (43), (44) y (45)

$$
\left|A^{\beta} u^{\prime \prime}\right|^{2}+\left|A^{\alpha+\beta+\gamma / 2} u^{\prime}\right|^{2}+\left|A^{\alpha+\beta+\gamma} u\right|^{2} \leq k^{2},
$$

lo que demuestra el teorema 1.

PROBLEMA NO-LINEAL. En esta parte demostraremos la existencia de solución del problema no-lineal

$$
\begin{aligned}
& u^{\prime \prime}+M\left(\left|A^{\alpha} u(t)\right|^{2},\left|A^{\beta} u^{\prime}(t)\right|^{2}\right) A^{\gamma} u=f \\
& u(0)=u_{0} ; u^{\prime}(0)=u_{1}
\end{aligned}
$$

utilizando una técnica de aproximaciones sucesivas aplicado al conjunto G definido anteriormente.

En las condiciones establecidas en el teorema 1, se tiene que para todo $v \in G$ existe una única solución del problema

$$
\begin{aligned}
& u^{\prime \prime}+M\left(\left|A^{\alpha} v(t)\right|^{2},\left|A^{\beta} v^{\prime}(t)\right|^{2}\right) A^{\gamma} u=f \\
& u(0)=u_{0} ; u^{\prime}(0)=u_{1}
\end{aligned}
$$


La idea es resolver una sucesión de problemas de la forma

$$
\begin{aligned}
& z_{p}^{\prime \prime}+M\left(\left|A^{\alpha} z_{p-1}(t)\right|^{2},\left|A^{\beta} z_{p-1}^{\prime}(t)\right|^{2}\right) A^{\gamma} z_{p}=f \\
& z_{p}(0)=u_{0} ; z_{p}^{\prime}(0)=u_{1} \\
& P \geq 2
\end{aligned}
$$

donde $z_{2}$ es la única solución del problema,

$$
\begin{aligned}
& z_{2}^{\prime \prime}+M\left(\left|A^{\alpha} u_{0}\right|^{2},\left|A^{\beta} u_{1}\right|^{2}\right) A^{\gamma} z_{2}=f \\
& z_{2}(0)=u_{0} ; z_{2}^{\prime}(0)=u_{1} .
\end{aligned}
$$

Desde el teorema 1 podemos definir una función $S: G \rightarrow G$ tal que $S z_{p-1}=z_{p}, \quad p \geq 3$, donde $z_{p}$ es la única solución del problema (53).

La dificultad principal es demostrar la convergencia

$$
N\left(z_{p}\right) A^{\gamma} z_{p} \stackrel{m \rightarrow \infty}{\longrightarrow} N(z) A^{\gamma} z \text { en } L^{2}\left(0, T_{0} ; D\left(A^{\beta}\right)\right)
$$

donde

$$
N\left(z_{p}\right)=M\left(\left|A^{\alpha} z_{p-1}\right|^{2},\left|A^{\beta} z_{p-1}^{\prime}\right|^{2}\right) ; N(z)=M\left(\left|A^{\alpha} z\right|^{2},\left|A^{\beta} z^{\prime}\right|^{2}\right)
$$

En primer lugar probaremos que,

$$
N\left(z_{p}\right) \rightarrow N(u) \text { fuerte en } L^{2}\left(0, T_{0} ;\right) .
$$

En efecto, la sucesión $\left(z_{p}\right)_{p \geq 2} \subset G$ y con argumentos de compacidad similares a los utilizados en la demostración del Teorema 1, obtenemos que existe una función

$$
\begin{aligned}
& z \in L^{\infty}\left(0, T_{0} ; D\left(A^{\alpha+\beta+\gamma}\right)\right) \text { tal que } \\
&\left|A^{\alpha} z_{p}\right|^{2} \stackrel{m \rightarrow \infty}{\longrightarrow}\left|A^{\alpha} z\right|^{2} \text { fuerte en } L^{2}\left(0, T_{0}\right) \\
&\left|A^{\beta} u_{p}^{\prime}\right|^{2} \stackrel{m \rightarrow \infty}{\longrightarrow}\left|A^{\beta} u^{\prime}\right|^{2} \text { fuerte en } L^{2}\left(0, T_{0}\right) .
\end{aligned}
$$

Ahora, por la hipótesis $\mathrm{H}_{1}$ sobre la función $\mathrm{M}$

$$
\begin{aligned}
& M\left(s_{1}, r_{1}\right)-M\left(s_{2}, r_{2}\right)=M_{s}\left(\theta_{1}, r_{1}\right)\left(s_{1}-s_{2}\right)+M_{s}\left(s_{1}, \theta_{2}\right)\left(r_{1}-r_{2}\right) \\
& \quad \forall s_{1}, s_{2}, r_{1}, r_{2} \in R, \theta_{1} \in\left[s_{1}, s_{2}\right], \theta_{2} \in\left[r_{1}, r_{2}\right] .
\end{aligned}
$$


Entonces,

$$
\begin{aligned}
& \int_{0}^{T_{0}}\left|M\left(\left|A^{\alpha} z_{p-1}(t)\right|^{2},\left|A^{\beta} z_{p-1}^{\prime}(t)\right|\right)-M\left(\left|A^{\alpha} z(t)\right|^{2},\left|A^{\beta} z^{\prime}(t)\right|\right)\right|^{2} d t \leq \\
& \leq \int_{0}^{T_{0}}\left|M_{s}\left(\theta_{1},\left|A^{\beta} z_{p-1}^{\prime}(t)\right|\right)\right|\left|A^{\alpha} z_{p-1}(t)\right|^{2}-\left|A^{\alpha} z(t)\right|^{2} \|^{2} d t+ \\
& +\int_{0}^{T_{0}}\left|M_{r}\left(\left|A^{\alpha} z_{p-1}(t)\right|, \theta_{2}\right)\right|\left|A^{\beta} z_{p-1}^{\prime}(t)\right|^{2}-\left.\left|A^{\beta} z^{\prime}(t)\right|^{2}\right|^{2} d t \leq \\
& \leq C \int_{0}^{T_{0}}\left\|\left.|| A^{\alpha} z_{p-1}(t)\right|^{2}-\left|A^{\alpha} z(t)\right|^{2}\right\|^{2} d t+ \\
& +C \int_{0}^{T_{0}}\left\|\left|A^{\beta} z_{p-1}^{\prime}(t)\right|^{2}-\left|A^{\beta} z^{\prime}(t)\right|^{2}\right\|^{2} d t \stackrel{p \rightarrow \infty}{\longrightarrow} 0 .
\end{aligned}
$$

Luego,

$$
\begin{aligned}
& \int_{0}^{T_{0}}\left|N\left(z_{p-1}(t)\right)\left(A^{\gamma} z_{p}(t), w(t)\right)-N(z(t))\left(A^{\gamma} z(t), w(t)\right)\right| d t= \\
& \leq \int_{0}^{T_{0}}\left|N\left(z_{p-1}(t)\right)\right|\left|A^{\gamma} z_{p-1}(t)-A^{\gamma} z(t)\right||w(t)| d t+ \\
& +\int_{0}^{T_{0}}\left|N\left(z_{p-1}(t)\right)-N(z(t))\right|\left|A^{\gamma} z(t)\right||w(t)| d t \stackrel{p \rightarrow \infty}{\longrightarrow} 0 .
\end{aligned}
$$

Utilizando (62) y (63), obtenemos finalmente que la función $z$ verifica la ecuación (49).

\section{UNICIDAD}

Sean $u, z$ dos soluciones de (49) y (50). Entonces se verifica que

$$
\begin{aligned}
& u, z \in L^{\infty}\left(0, T_{0} ; D\left(A^{\alpha+\beta+3 \gamma / 2}\right)\right) \\
& u^{\prime}, z^{\prime} \in L^{\infty}\left(0, T_{0} ; D\left(A^{\alpha+\beta+\gamma}\right)\right) \\
& u^{\prime \prime}, z^{\prime \prime} \in L^{\infty}\left(0, T_{0} ; D\left(A^{\alpha+\beta+\gamma / 2}\right)\right) \\
& u(0)=z(0)=u_{0} \\
& u^{\prime}(0)=z^{\prime}(0)=u_{1}
\end{aligned}
$$

Sea, $y=u-z$. Entonces, 


$$
\begin{aligned}
& y \in L^{\infty}\left(0, T_{0} ; D\left(A^{\alpha+\beta+3 \gamma / 2}\right)\right) \\
& y^{\prime} \in L^{\infty}\left(0, T_{0} ; D\left(A^{\alpha+\beta+\gamma}\right)\right) \\
& y^{\prime \prime} \in L^{\infty}\left(0, T_{0} ; D\left(A^{\alpha+\beta+\gamma / 2}\right)\right) \\
& y(0)=0 \\
& y^{\prime}(0)=0
\end{aligned}
$$

satisface la ecuación

$$
\left(y^{\prime \prime}(t), w\right)+N(u(t))\left(A^{\gamma} y(t), w\right)=(N(z(t))-N(u(t)))\left(A^{\gamma} z(t), w\right)
$$

Haciendo $v=A^{2 \beta} y^{\prime}(t)$ en (74) obtenemos,

$$
\begin{aligned}
\frac{d}{d t}\left\{\left|A^{\beta} y^{\prime}(t)\right|^{2}+N(u(t))\left|A^{\beta+\gamma / 2} y(t)\right|^{2}\right\}= & (N(z(t))-N(u(t)))\left(A^{\beta+\gamma / 2} z(t), A^{\beta} y^{\prime}(t)\right) \\
& +N^{\prime}(u(t))\left|A^{\beta+\gamma / 2} y(t)\right|^{2} .
\end{aligned}
$$

Tenemos,

$$
\begin{aligned}
& \mid M\left(\left|A^{\alpha} z(t)\right|^{2},\left|A^{\beta} z^{\prime}(t)\right|\right)-M\left(\left|A^{\alpha} u(t)\right|^{2},\left|A^{\beta} u^{\prime}(t)\right|||\right. \\
& \leq\left|M_{s}\left(\theta_{1},\left|A^{\beta} z^{\prime}(t)\right|\right)\right|\left|A^{\alpha} z(t)\right|^{2}-\left|A^{\alpha} u(t)\right|^{2}|| \\
& +\left|M_{r}\left(\left|A^{\alpha} z(t)\right|, \theta_{2}\right)\right|\left|A^{\beta} z^{\prime}(t)\right|^{2}-\left|A^{\beta} u^{\prime}(t)\right|^{2}|| \\
& \leq C|| A^{\alpha} z(t)|+| A^{\alpha} u(t)|||| A^{\alpha} z(t)|-| A^{\alpha} u(t)|| \\
& +C|| A^{\beta} z^{\prime}(t)|+| A^{\beta} u^{\prime}(t)|||| A^{\beta} z^{\prime}(t)|-| A^{\beta} u^{\prime}(t) \| \\
& \leq C\left\{\left|A^{\alpha} y(t)\right|+\left|A^{\beta} y^{\prime}(t)\right|\right\} .
\end{aligned}
$$

Reemplazando en (75) obtenemos

$$
\begin{aligned}
\frac{d}{d t}\left\{\left|A^{\beta} w^{\prime}(t)\right|^{2}+N(u(t))\left|A^{\beta+\gamma / 2} w(t)\right|^{2}\right\} & \leq C\left\{\left|A^{\alpha} w(t)\right|+\left|A^{\beta} w^{\prime}(t)\right|\right\}\left|A^{\beta} w^{\prime}(t)\right| \leq \\
& \leq C\left|A^{\alpha} w(t)\right|^{2}+C\left|A^{\beta} w^{\prime}(t)\right|^{2} \leq \\
& \leq C\left|A^{\beta+\gamma / 2} w(t)\right|^{2}+C\left|A^{\beta} w^{\prime}(t)\right|^{2}
\end{aligned}
$$

Integrando de 0 a $t$ y teniendo en cuenta que $w(0)=w^{\prime}(0)=0$ obtenemos 


$$
\eta(t)=\left|A^{\beta} w^{\prime}(t)\right|^{2}+d_{0}\left|A^{\beta+\gamma / 2} w(t)\right|^{2} \leq C \int_{0}^{t} \eta(s) d s
$$

y por el lema de Gronwall $A^{\beta} w^{\prime}(t)=A^{\beta+\gamma / 2} w(t)=0 \quad \forall t \in\left[0, T_{0}\right]$. Luego $u=z$.

\section{BIBLIOGRAFÍA}

1. Alves de Lima, O., Oliveira M. "Global solutions for small data of the Carrier equation with dissipative term ." Atas do $46^{\circ}$ Seminario Brasileiro de Análise (1997).

2. Arosio, A., Spagnolo S. "Global solutions of the Cauchy problem for a non-linear Hyperbolic Equation". Universita di Pisa . Departamento de Matemática. Roma (1982).

3. Carrier, G.F. "On the non-linear vibration problem of the elastic string" Quart. Appl. Math. - 3 - (1945).

4. Cousin A.; Frota C.; Larkin N.; Medeiros, L.A. "On the abstrac model of KirchhoffCarrier Equation". Comm. In App. Analysis. - 3 - (1997).

5. Crippa H. "On Local Solutions of Some mildly Degenerate Hyperbolic Equations". Non-linear Analysis, Vol. 21 (8) (1993).

6. Ebihara, Y. Medeiros, L. A. Milla. M. "Local solutions for a nonlinear degenerate hyperbolic equations". Nonlinear Analysis.Vol.10. (1986).

7. Ikehata, R. "A note on the global solvability solutions on some nonlinear wave equations with dissipative term". Diff. Int. Equ. 8 (1995).

8. Izaguirre, R. - Véliz, V. "Solución local para una clase de ecuaciones no-lineales degeneradas tipo Kirchhoff-Carrier". I Seminario Internacional de Ecuaciones Diferenciales y Aplicaciones. Universidad Ricardo Palma - Lima -Perú- (1999).

9. Kirchhoff G. "Vorlesungenuber mechanik". Teubner, Leipzig (1883).

10. Lions, J.L. “Quelques Méthodes de Resolution des Problemes aux limites nonlineares". Dunod. Paris.(1969).

11. Medeiros, L. A., Milla M. "Solutions for the Equation of Nonlinear Vibrations Sobolev Spaces of Fractionary Order". Math. Apl. Comp. 6 (1987).

12. Medeiros, L.A., Milla M. "Remarks on a nonlinear model vibrations of string with damping". $30^{\circ}$ Seminario Brasileiro de Análise.L.N.C.C.-R.J.(1989).

13. Nakao M. ,Ono K. "Existence of Global Solutions to the Cauchy problem for the semilinear dissipative wave equations". Math. Z. 214 (1993). 
14. Nishihara A. ,Yamada Y. " On global solutions for some degenerate quasilinear hyperbolic equations with dissipative term". Funkcialaj Ekvacioj 33 (1990).

15. Ono, K. "On Global existence,asymptotic stability and blowing up solutions for some degenerate nonlinear wave equations of Kirchhoff type with a strong disssipation". Math. Meth. Apll. Sci. 20 (1997).

16. Ono, K. "Global existence, Decay and Blowup of Solutions for Some Mildly Degenerate Nonlinear Kirchhoff String". J. Diff. Eq. 137 (1997).

17. Perla, G. "On classical solutions of a quasilinear hyperbolic equations". Nonlinear Analysis. Vol. 3 (1979).

18. Pohozaev, S. "The Kirchoff quasilinear hyperbolic equation". Differential Equations Vol. 21. (1985).

19. Pohozaev S. "On a class of quasilinear hyperbolic equations". Math. Sbornik, Vol. 96 (1975).

20. Rivera, P. "On local strong solutions of a nonlinear partial differential equation". Appl. Analysis. Vol. 10. (1980).

21. Yamada, Y. "Some Nonlinear Degenerate Wave Equations". Non Linear Analysis, 10 (11) (1987). 